\title{
Germanica
}

\section{Arthur Schnitzler et l'Autriche dans La Revue de Genève, 1920-1930}

Arthur Schnitzler and Austria in La Revue de Genève, 1920-1930

Arthur Schnitzler und Oesterreich in La Revue de Genève, 1920-1930

Jacques Le Rider

\section{(2) OpenEdition}

Journals

Édition électronique

URL : http://journals.openedition.org/germanica/2117

DOI : $10.4000 /$ germanica. 2117

ISSN : 2107-0784

Éditeur

Université de Lille

Édition imprimée

Date de publication : 15 juin 2013

Pagination : 25-36

ISBN : 9782913857315

ISSN : 0984-2632

\section{Référence électronique}

Jacques Le Rider, «Arthur Schnitzler et l'Autriche dans La Revue de Genève, 1920-1930 », Germanica

[En ligne], 52 | 2013, mis en ligne le 20 juin 2013, consulté le 06 octobre 2020. URL : http:// journals.openedition.org/germanica/2117 ; DOI : https://doi.org/10.4000/germanica.2117

(c) Tous droits réservés 


\title{
Arthur Schnitzler et l'Autriche dans La Revue de Genève, 1920-1930
}

\author{
Jacques LE RIDER \\ EPHE (École Pratique des Hautes Études)
}

En introduction d'une contribution consacrée à la présence d'Arthur Schnitzler et de l'Autriche dans La Revue de Genève entre 1920$1930^{1}$, il est bon de rappeler que la «modernité viennoise », comme on est convenu de l'appeler - même si l'homogénéité suggérée par le substantif Wiener Moderne masque des clivages non négligeables entre « modernes classiques » (de Hofmannsthal à Schnitzler) et « modernes de rupture » (de Karl Kraus aux premiers expressionnistes) -, arrive parfois en France au terme de certaines médiations. La Belgique francophone et la Suisse romande sont des aires culturelles accoutumées au dialogue entre culture de langue allemande et culture française. Rappelons l'importance du Palais Stoclet à Bruxelles, la principale réalisation d'un Gesamtkunstwerk architectural et décoratif viennois en terre étrangère, et l'importance de la Belgique chez Stefan Zweig, du moins jusqu'à la Première Guerre mondiale. En ce qui concerne la Suisse, je rappellerai les intéressantes réflexions de Hofmannsthal sur le contexte interculturel d'Amiel. La Première Guerre mondiale a considérablement accru l'importance de la Suisse, et pas seulement de la Suisse romande, comme terre de refuge et de rencontres européennes pour les écrivains et les artistes allemands et autrichiens.

1. - Cf. sur la question de la réception de Schnitzler, l'ouvrage de Karl Zieger: Arthur Schnitzler et la France, 1894-1938. Enquête sur une réception, Villeneuve d'Ascq, Presses universitaires du Septentrion, 2012, qui livre un panorama exhaustif. 


\section{Robert de Traz et La revue de Genève}

C'est dans le contexte de l'après-Grande Guerre que naît La Revue de Genève. Le premier directeur de la rédaction, Robert de Traz, né en 1884 à Paris, d'un père vaudois et d'une mère française, a trouvé chez Barrès, à vingt ans, la confirmation de sa conscience helvétique: "Plus j'étais barrésien, plus je me sentais suisse $»^{2}$, écrira-t-il plus tard. De 1911 à 1913, il a dirigé la revue Feuillets, imprégnée d'idéologie maurrassienne, d'antilibéralisme, d'antiparlementarisme, d'anticapitalisme, de xénophobie, le tout mis au service d'un « helvétisme » qu'on hésite à appeler nationaliste puisque Robert de Traz plaide pour la cohésion des Romands et des Alémaniques.

Pendant la Première Guerre mondiale, il milite en particulier contre Romain Rolland. Celui-ci écrit dans son Journal des années de guerre 1914-1919:

Dans la Semaine littéraire du 3 novembre [1917], Robert de Traz consacre un long article à l'influence néfaste des «pacifistes français réfugiés en Suisse » et principalement à la mienne sur la jeunesse suisse. Il me traite de « faux Christ des nations », et attribue mon pacifisme à une vanité d'écrivain, qui préfère son Jean-Christophe à la France, « l'idéalisme international » de l'un à « l'idéalisme patriotique » de l'autre ${ }^{3}$.

Au lendemain de la Grande Guerre, Robert de Traz voudra que $L a$ Revue de Genève soit « internationale, sans être internationaliste ${ }^{4}$. Son cosmopolitisme européen sera fidèle à l'idée nationale. Par patriotisme suisse, il soutient la création de la Société des Nations ; fin 1920, il écrit :

Wilson a donné à la tradition genevoise la plus éclatante consécration qui soit. C'est en considération de la Réforme, de Rousseau, de la Croix-Rouge que Wilson a imposé Genève à des gens dont beaucoup la détestent, et eussent bien voulu lui ravir cet extraordinaire privilège 5 .

Il condensera son « mythe de Genève » dans le livre intitulé L'Esprit de Genève publié en 1929. À partir de 1922, Robert de Traz participe

2. - Cité in Landry Charrier : "La Revue de Genève », les relations franco-allemandes et l'idée d'Europe unie (1920-1925), Genève, Slatkine Érudition, 2009, p. 15.

3. - Romain Rolland, Journal des années de guerre 1914-1919. Notes et documents pour servir à l'histoire morale de l'Europe de ce temps, Paris, Albin Michel, 1952, p. 1338.

4. - Cité in Landry Charrier, "La Revue de Genève », les relations franco-allemandes et l'idée d'Europe unie, op. cit., p. 20. Cf. Jean-Pierre Meylan, La Revue de Genève, miroir des lettres européennes (1920-1930), Genève, Droz, 1969.

5. - Cité ibid., p. 23. 
aux décades de Pontigny où il rencontre bon nombre des auteurs qu'il publie dans La Revue de Genève. C'est dans cet esprit qu'il anime sa revue: international, mais non internationaliste; cosmopolite, mais fidèle à l'idée de l'Europe des nations; suisse dans son esprit, mais non restreint à la défense et illustration de la culture romande. Il conserve un réseau parisien qui soutient La Revue de Genève: on peut mentionner Edmond Jaloux dont l'admiration pour le romantisme allemand et le rôle d'introducteur de la littérature allemande et autrichienne contemporaine en France est bien connu; Jean Cassou, qui publie en 1926 Les Harmonies viennoises; Jean Mistler, qui publie le roman Châteaux en Bavière en 1925, la Vie d'E.T.A. Hoffmann en 1927 et une petite monographie intitulée Vienne en 1931.

L'influence de Robert de Traz sur les choix éditoriaux de la revue est déterminante de 1920 à $1924\left(n^{\circ} 1\right.$ de juillet 1920 à $n^{\circ} 53$ de novembre 1924), un peu moins grande à partir du moment où, en décembre 1924, La Revue de Genève fusionne avec la Bibliothèque universelle de Lausanne, dont l'histoire remonte à 1815. La revue s'appelle désormais Bibliothèque universelle et Revue de Genève et paraît de décembre 1924 à décembre 1930. Les difficultés financières de La Revue de Genève, dues en particulier aux coûts de la traduction de nombreuses contributions étrangères, expliquent ce mariage forcé avec la Bibliothèque universelle, publiée par les frères Payot. La revue lausannoise connaissait, elle aussi, des problèmes financiers. Elle suivait une ligne éditoriale plus régionale qu'internationale. À partir de 1925, Robert de Traz a du mal à défendre l'orientation internationale qu'il avait donnée à La Revue de Genève. En 1926, il est remplacé à la tête de la Bibliothèque universelle et Revue de Genève par Jacques Chenevière et la revue se « romandise » malgré la résistance opiniâtre de Robert de Traz. À la fin de 1927, la revue absorbe encore La Semaine littéraire, un hebdomadaire d'inspiration locale. Une lettre de Denis de Rougemont à Robert de Traz, du 10 janvier 1928, met à nu les divergences suscitées par ces fusions successives:

Pour mon compte, je ne suis pas du tout pour une romandisation de La Revue de Genève. Il faut qu'elle reste ce qu'elle a été jusqu'ici, c'était très bien, et je ne vois pas en quoi l'invasion des Romands pourrait hausser son niveau, au contraire...6

Depuis le début, La Revue de Genève a dû affronter de sérieuses difficultés financières. La dévaluation de la plupart des monnaies européennes et la relative stabilité du franc suisse rendait la diffusion de la revue à l'étranger difficile. La consolidation permise par la fusion avec

6. - Cité ibid., p. 56. 
La Bibliothèque Universelle fut compromise par les dissensions entre les pôles genevois et lausannois. En 1930, la revue pouvait compter sur un lectorat de deux mille personnes environ, mais elle ne conservait plus que cent trente-trois abonnés en Suisse. Durant une decennia, La Revue de Genève a cependant joué un rôle important dans les échanges intellectuels européens ${ }^{7}$.

La conviction qui inspire Robert de Traz au moment du lancement de La Revue de Genève est que " l'instauration d'une paix durable ne peut résulter que d'une connaissance accrue de la culture de ses voisins » et il « accorde une place prédominante aux nouvelles et romans, aux études de caractère général ainsi qu'aux textes de critique, d'histoire ou de politique, sans autre intermédiaire que la traduction $»^{8}$. Robert de Traz n'a pas le goût des avant-gardes, mais il obtient de prestigieuses collaborations. Du côté français et suisse romand: Barrès, Mauriac, Montherlant, Edmond Jaloux, Gide, Proust, Giraudoux, Valery Larbaud, Ramuz. La Revue de Genève publie en juillet 1925 un article de Denis de Rougemont et en janvier 1929, un essai de Marguerite Yourcenar intitulé « Diagnostic de l'Europe ». Dans le domaine allemand, Heinrich et Thomas Mann, Rilke, Hesse, Hofmannsthal, Gerhart Hauptmann, mais aussi Wassermann, Sternheim, Werfel, Stefan Zweig sont publiés dans la revue. Sans oublier Arthur Schnitzler.

À travers une liste qui parlera aux « austriacistes », rappelons les textes publiés, dans La Revue de Genève, de Hofmannsthal: Beethoven (juin 1921) et La Femme sans ombre (de juin à août 1930)9 ${ }^{9}$; de Rilke: Sur des CEuvres de Rodin (mai 1925) ${ }^{10}$ et Quatrains valaisans (avril 1926); de Werfel: Poèmes (avril 1924)11; de Stefan Zweig: Hölderlin (décembre 1927) ${ }^{12}$. Et soulignons la curiosité que constitue

7. - Cf. Landry Charrier, op. cit., chapitre 1-2-3, « Biographie financière de $L a$ Revue de Genève », en particulier p. 62-63.

8. - Ibid., p. 31 .

9. - Cf. aussi le compte rendu par Marcel Beaufils de Hugo von Hofmannsthal, La Tour, Berlin, Fischer, in Bibliothèque universelle et Revue de Genève, 1928, vol. 2 (juillet-décembre), p. 1419 sq. (très critique : « accessoires tragiques éculés ; effets d'avance émoussés et qui sentent le moisi ; pathos schillérien qui frise à chaque instant la réminiscence »).

10. - Trad. René Rapin, in Bibliothèque universelle et Revue de Genève, 1925, vol. 1 (janvier-juin), p. 527-541. Cf. René Rapin, « Rilke, Jacobsen et Rodin », in Bibliothèque universelle et Revue de Genève, 1925, vol. 2 (juillet-décembre), p. 17341743 .

11. - Cf. aussi le compte rendu par Marcel Beaufils de Franz Werfel, Der Abituriententag, Leipzig, Paul Zsolnay, in Bibliothèque universelle et Revue de Genève, 1929, vol. 1 (janvier-juin), p. 124 sq.

12. - Stefan Zweig, « Hölderlin », trad. Alzir Hella et Olivier Bournac, in Bibliothèque universelle et Revue de Genève, 1927, vol. 2 (juillet-décembre), p. 653-667, accompagné d'une «Remarque » à propos de Stefan Zweig (indications biographiques), 
la présence de Paul Zifferer, dont La Revue de Genève publie en juillet 1925 L'Auberge engloutie $^{13}$. Ce texte était accompagné d'une étude de Marcel Dunan ${ }^{14}$ intitulée "Paul Zifferer, romancier autrichien » qui présentait cet auteur comme l'illustration de "l'originalité et de l'autonomie de la "littérature autrichienne" au sein du Reich de la langue allemande ». La réputation de Zifferer (1879-1928), poeta minor du Jung Wien aujourd'hui oublié, est grande à l'époque : comme rédacteur de la Neue Freie Presse, il a rendu compte de nouveautés littéraires françaises au public viennois. Après la guerre, il s'est établi à Paris comme conseiller littéraire de l'Ambassade d'Autriche. Il sert d'intermédiaire pour les échanges littéraires. C'est lui qui organise le voyage à Paris de Hofmannsthal en 192515. L'Auberge engloutie de Zifferer est publiée dans le même volume de la Bibliothèque universelle et Revue de Genève que «Les morts se taisent » de Schnitzler, mais c'est Zifferer qui fait l'objet d'un essai de présentation, alors qu'aucune étude n'est consacrée à Schnitzler. Pour en savoir plus sur ce dernier, le lecteur doit se contenter du passage de l'essai de Dunan sur Zifferer qui évoque en ces termes le genre de la nouvelle:

p. 786 ; Cf. aussi le compte rendu par Robert de Traz d'Amok et Balzac, Dickens, de Stefan Zweig, traduits par Alzir Hella et Olivier Bournac, publiés à Paris chez Simon Kra, très élogieux ( «Ce qui est curieux et admirable, c'est de nous les montrer [Balzac et Dickens] dans le rythme synthétique et saccadé de la vie »), in Bibliothèque universelle et Revue de Genève, 1927, vol. 1 (janvier-juin), p. 831; le compte rendu par Marcel Beaufils de Stefan Zweig, Trois poètes de leur propre vie : Casanova -Stendhal-Tolstoï, Leipzig, Insel, in Bibliothèque universelle et Revue de Genève, 1928, vol. 2 (juilletdécembre), p. 966 sq. (très critique à propos de l'essai sur Tolstoï : "L'impartialité manque. À chaque page tombe un mot malheureux ») ; le compte rendu par Marcel Beaufils de Stefan Zweig, Hölderlin, trad. A. Hella et O. Bournac, Stock, in Bibliothèque universelle et Revue de Genève, 1929, vol. 1 (janvier-juin), p. 121 ; le compte rendu par Daniel-Rops de Stefan Zweig, Vingt-quatre heures dans la vie d'une femme, trad. A. Hella et O. Bournac, Attinger, in Bibliothèque universelle et Revue de Genève, 1929, vol. 2 (juillet-décembre), p. 791.

13. - Trad. Marcel Dunan. Bibliothèque universelle et Revue de Genève, 1925, vol. 2 (juillet-décembre), p. 798-811.

14. - L'historien Marcel Dunan (1885-1978), professeur d'histoire de la Révolution et de l'Empire à la Sorbonne de 1946 à 1955, est dans l'entre-deux-guerres un expert de la question autrichienne. Il publie en 1921, chez Rieder à Paris, L'Autriche, un livre de 132 pages, dans lequel il est présenté comme l'envoyé spécial du Temps à Vienne (ouvrage traduit en allemand sous le titre Österreich, chez Manz à Vienne en 1922). Il traduit de Raoul Auernheimer, Le Marchand de secrets (Plon-Nourrit, 1924), et de Paul Zifferer, La Ville impériale, 1924. De 1926 à 1931, il est le premier directeur du Centre de Hautes études françaises à Vienne (devenu Institut français de Vienne et rattaché à la Sorbonne à partir de 1931), installé de 1926 à 1931 Bäckerstrasse 13 dans le 1er arrondissement. En 1934, il publie encore la brochure de 103 pages Le Problème autrichien, Paris, Le Redressement français, 1934 (Cahiers du redressement français, 2e série, vol. 13).

15. - Jean-Pierre Meylan, op. cit., p. 345. 
La nouvelle, ce genre littéraire aujourd'hui trop méconnu chez nous, y passe pour répondre au talent des auteurs de souffle court et d'imagination pauvre. La littérature autrichienne lui fait à juste titre une plus large place, et peut-être une place privilégiée, puisque leurs nouvelles ont consacré la réputation d'écrivains de ces deux éminents auteurs dramatiques, Arthur Schnitzler et Raoul Auernheimer. Dans ce genre, Paul Zifferer s'est fait une place à part en adoptant un type original de volumes. Ses recueils de nouvelles ne sont pas formés de pièces détachées mais de récits groupés pour constituer un tout ${ }^{16}$.

En janvier 1923, La Revue de Genève traite de «La situation des intellectuels » en Autriche. Cette « chronique internationale », signée de Hans Sperl, «président du Conseil central des travailleurs intellectuels en Autriche », fait un tableau fort noir de la « misère de l'Autriche, telle qu'elle s'est développée depuis $1914 »$. Certaines professions intellectuelles (médecins, avocats, ingénieurs, notaires, écrivains, précise Hans Sperl) affrontent les pires difficultés matérielles; les architectes, « se trouvent réduits à l'impuissance totale »; la misère sévit dans les laboratoires de recherche scientifique, le dénuement paralyse les hôpitaux, la pauvreté interdit aux bibliothèques de développer leurs fonds ${ }^{17}$. Au deuxième semestre 1923, La Revue de Genève publie une information intitulée «Musique autrichienne » qui annonce que le festival organisé par l'Union intellectuelle autrichienne au Mozarteum de Salzbourg, du 8 au 11 août, remplacera cette année « le grand Festival de Salzbourg »; au programme: une conférence de Richard Specht et des œuvres de Korngold, Schönberg, Richard Strauss, Zemlinsky, etc. ${ }^{18}$.

La traduction française de textes d'auteurs autrichiens est parfois accompagnée d'un dossier consacré à l'identité culturelle autrichienne. Ainsi la traduction par Alexandre Vialatte du conte en prose La Femme sans ombre, de Hofmannsthal, dans le numéro du premier semestre $1930^{19}$, voisine avec un article d'Erwin Rieger intitulé « Hofmannsthal, l'Autrichien », qui commente l'ouvrage de Geneviève Bianquis $L a$ Poésie autrichienne de Hofmannsthal à Rilke et avec un texte du comte Alexandre Hoyos intitulé « Le rattachement de l'Autriche à l'Alle-

16. - Marcel Dunan, " Paul Zifferer, romancier autrichien », in Bibliothèque universelle et Revue de Genève, 1925, vol. 2 (juillet-décembre), p. 792-797, citation p. $795 \mathrm{sq}$.

17. - Hans Sperl, « Chronique internationale - Autriche - La situation des intellectuels », in La Revue de Genève, n³1, janvier 1923, p. 66-75.

18. - «Musique autrichienne », in La Revue de Genève, tome VII, juilletdécembre 1923, p. 259 sq.

19. - La deuxième partie de La Femme sans ombre est publiée dans le numéro suivant, Bibliothèque universelle et Revue de Genève, 1930, vol. 2 (juillet-décembre), p. 197-223. 
magne $»^{20}$. L'une et l'autre étude insistent sur la difficulté de dissocier la culture autrichienne de la culture allemande, ce qui tranche sur les discours savants français à propos de l'Autriche qui, dans les années 1920-1938, insistent au contraire sur l'irréductible singularité de la culture autrichienne. Erwin Rieger estime que Hofmannsthal « proclame la mission allemande et européenne de l'Autriche et de l'Autrichien » et que, « au-delà d'une civilisation fondée essentiellement sur la tradition, [il] rêve d'une littérature mondiale dans le sens où Goethe l'a conçue ${ }^{21}$. Alexandre Hoyos conclut pour sa part, faisant allusion à la guerre austro-prussienne de 1866:

Jamais plus, à vues humaines, l'Autriche n'approuvera une politique hostile au Reich allemand: jamais plus elle ne se ralliera à un groupement qui poursuivrait des buts anti-allemands. Le sentiment d'une commune origine et les souvenirs d'une histoire commune demeurent trop vivaces dans tous les milieux autrichiens ${ }^{22}$.

En 1928, une chronique d'Edmond Rossier considérait que « la conviction progermanique semble avoir gagné, non seulement la majorité du peuple viennois, mais le pays dans son ensemble, jusqu'au Tirol (sic) et au Vorarlberg »23. Le volume 2 de 1929 contient une étude d'Erwin Rieger sur «Vienne », qui insiste cette fois sur « le caractère non allemand $» 24$ de la capitale autrichienne. Le volume 2 de 1930 publie une nouvelle chronique d'Erwin Rieger, intitulée « Barbarie villageoise et raffinements viennois », qui évoque l'esprit de guerre civile qui, selon lui, l'emporte en Autriche sur le sentiment national: "L'Autrichien est en premier lieu Sozialdemokrat ou Heimwehrmann » ${ }^{25}$. Après avoir vanté la «droiture de caractère » de Schober, hommage qui étonnera

20. - Hugo von Hofmannsthal, « La Femme sans ombre », in Bibliothèque universelle et Revue de Genève, 1930, vol. 1 (janvier-juin), p. 657-679; Erwin Rieger, «Les chroniques nationales - Autriche - Hofmannsthal, l'Autrichien », ibid., p. 368375 ; comte Alexandre Hoyos « Le rattachement de la confédération autrichienne à l'Allemagne » (le titre courant et le titre mentionné dans la table des matières est: « Le rattachement de l'Autriche à l'Allemagne »), ibid., p. 426-434.

21. - Ibid., p. 372 sq.

22. - Ibid., p. 434.

23. - Edmond Rossier, « La chronique internationale - Autriche et Allemagne », in Bibliothèque universelle et Revue de Genève, 1928, vol. 2 (juillet-décembre), p. 12671274, citation p. 1272.

24. - Erwin Rieger, "Les chroniques nationales - Autriche - Vienne », in Bibliothèque universelle et Revue de Genève, 1929, vol. 2 (juillet-décembre), p. 599607, citation p. 605.

25. - Erwin Rieger, « Les chroniques nationales - Autriche - Barbarie villageoise et raffinements viennois », in Bibliothèque universelle et Revue de Genève, 1930, vol. 2 (juillet-décembre), p. 628-633. Citation p. 630. 
les lecteurs de Karl Kraus, Erwin Rieger insiste sur l'importance des « écrivains, artistes, savants » juifs à Vienne :

Arthur Schnitzler, par exemple, Jakob Wassermann, Karl Kraus, Stefan Zweig, Freud, Max Reinhardt (et même Hofmannsthal a eu sa part de ce sang oriental. Notons que presque toutes les grandes figures de la Sozialdemokratie autrichienne, un Otto Bauer ou un Friedrich Adler, sont dans le même cas) ${ }^{26}$.

\section{Arthur Schnitzler et La Revue de Genève}

Venons-en à Schnitzler. De cet auteur, La Revue de Genève publie en novembre 1925 la nouvelle Les Morts se taisent, traduite par Geneviève Maury ${ }^{27}$. Cette germaniste, conseillère de La Revue de Genève pour le domaine allemand, est traductrice de Thomas Mann et de Hermann Hesse en particulier. Dans La Revue de Genève, elle a publié «L'Allemagne et la démocratie d'après les idées de Thomas Mann » en février 1921; «Le comte Hermann Keyserling », en mars 1922; un ensemble de documents à propos de la République de Weimar en janvier 1923, sous le titre « De la République allemande »; enfin "Stefan George », en janvier 1924; elle publiera un compte rendu de Geneviève Bianquis, La poésie autrichienne de Hofmannsthal à Rilke, en 1926, affirmant, sans plus de précisions, que « Mlle Geneviève Bianquis fait excellemment ressortir les caractères communs de cette poésie autrichienne, très différente de la poésie allemande ${ }^{28}$. Originaire de Vevey, Geneviève Maury, née en 1886, avait passé une grande partie de sa jeunesse en France, à Paris et en Haute-Vienne. Romain Rolland, un ami de la famille, portait un jugement très favorable sur les contes limousins de Geneviève Maury. Sa famille avait hérité de l'immense fortune du grand-père maternel, Jules Monnerat, qui avait participé au financement de la société de farine lactée fondée par Henri Nestlé. En 1933, Geneviève Maury épousera le chef d'orchestre Charles Munch ${ }^{29}$.

Évoquons maintenant l'autre manifestation importante de la présence de Schnitzler dans La Revue de Genève, le compte rendu de la réédition de Mademoiselle Else par Daniel-Rops paru dans le numéro de février 1930. Karl Zieger met en lumière les faiblesses de ce compte rendu, soulignant par exemple le caractère réducteur de la définition de

26. - Ibid., p. 631.

27. - Bibliothèque universelle et Revue de Genève, 1925, vol. 2 (juillet-décembre), p. $1824-1841$.

28. - Compte rendu par Geneviève Maury de «Geneviève Bianquis, La Poésie autrichienne... », in Bibliothèque universelle et Revue de Genève, 1926, vol. 2 (juilletdécembre), p. 534.

29. - Cf. Landry Charrier, La «Revue de Genève », les relations franco-allemandes et l'idée d'Europe unie, op. cit., p. 65 sq. 
Schnitzler comme auteur préférant « la longue nouvelle ou le roman bref » et les «drames de petites dimensions », mais aussi du verdict final: «Mademoiselle Else a de réelles qualités de mesure et de distinction, mais il faut bien dire aussi que [Schnitzler] manque de force $»^{30}$.

Sur ce point, on pourrait engager le débat avec Karl Zieger : le jugement « français » qui se condense dans ce compte rendu de Mademoiselle Else, sans doute représentatif de l'opinion la plus répandue en 1930, témoigne-t-il d'un aveuglement particulièrement prononcé dans la réception française - et suisse romande - de Schnitzler ou d'une idée reçue dans l'entre-deux-guerres, y compris en Allemagne? La réception française de Schnitzler, en tout cas dans la critique littéraire et parmi les germanistes européens, n'a-t-elle pas été caractérisée par une sous-évaluation de sa valeur littéraire? Il me semble que la tendance à placer Schnitzler au-dessous de Hofmannsthal et même, ce qui peut surprendre, en dessous de Stefan Zweig, dont la gloire littéraire est sans doute plus grande durant toute la première moitié $\mathrm{du} \mathrm{XX}^{\mathrm{e}}$ siècle, a persisté jusqu'à l'époque de la redécouverte et de la réévaluation de la « modernité viennoise », à partir des années 1970 et 1980. C'est depuis lors que les études schnitzlériennes ont acquis une envergure comparable aux études hofmannsthaliennes. Dans le cas particulier de Schnitzler, la disparition en 1981 de Suzanne Clauser (Dominique Auclères), l'intermédiaire incontournable de tous les projets éditoriaux schnitzlériens en France, a « radicalement changé la donne sur le plan éditorial [et] ouvert la voie à de nouvelles traductions $»^{31}$.

Malgré sa tiédeur et ses approximations, le compte rendu de DanielRops publié dans La Revue de Genève en février 1930 est probablement un bon résumé des points de vue français concernant Schnitzler à cette époque. Le jugement porté sur la technique du monologue intérieur, dans Fräulein Else, ne manque pas de pertinence, même s'il trahit des réticences, partagées au sein de la rédaction de La Revue de Genève, face au redoutable avant-gardisme de Joyce:

Le plus grand intérêt de cette histoire mince réside dans la technique qu'a employée l'auteur. Il a utilisé ce procédé de monologue intérieur que James Joyce et Larbaud mirent à la mode. L'emploi qu'en fait Schnitzler est, il faut le dire, très raisonnable, fort mesuré, et ce moyen lui permet d'analyser les sentiments de son héroïne avec une minutie et une profondeur attachantes ${ }^{32}$.

30. - Cf. Karl Zieger, Enquête sur une réception. Arthur Schnitzler et la France..., op. cit., p. 168 et 220 sqq.

31. - Ibid., p. 307.

32. - Cité in Karl Zieger, op. cit., p. 227 sq. 
Mais c'est surtout un autre passage du compte rendu de Daniel-Rops qui mérite une attention particulière :

On a distingué dans son œuvre, selon M. Félix Bertaux ${ }^{33}$, « une vibration freudienne, que dissimule à peine l'égalité de ton, une foncière et pathologique mélancolie qui donne une gravité au geste élégant. » [...] Tout cela est assez visible dans Mademoiselle Else, avec je ne sais quoi d'un peu affecté, voire d'artificiel, qui s'adapte assez bien à l'atmosphère de palace dans laquelle se déroule le récit, mais qui n'en paraît pas moins, en fin de compte, assez vain ${ }^{34}$.

\section{La Revue de Genève, la psychanalyse... et Schnitzler}

Cette allusion à la psychanalyse freudienne conduit à insister sur une spécificité de La Revue de Genève : cette revue a manifesté un intérêt tout particulier pour la psychanalyse et a contribué à son «transfert »dans l'aire culturelle française qui, dans l'entre-deux-guerres, reste réticente, surtout dans le milieu médical. Selon Jean-Pierre Meylan, « Robert de Traz est lui-même un fervent du freudisme ${ }^{35} »$; il prétend écrire ses romans dans l'esprit de la psychanalyse freudienne; dans l'Hommage à Marcel Proust (NRF, $\mathrm{n}^{\circ} 112$, janvier 1923), on trouve une "Note sur l'inconscient chez Marcel Proust » où Robert de Traz affirme que Proust a inventé une méthode d'analyse psychologique parallèle à celle de Freud, et, en 1923 également, il publie dans Les Nouvelles littéraires un texte intitulé «Visite à Freud » dans lequel il relate sa rencontre, lors d'un séjour à Vienne, avec le maître de la Berggasse 1936. Grâce à deux intermédiaires, Édouard Claparède et Oskar Pfister, il a obtenu de Freud l'autorisation de publier la première traduction française des Fünf Vorlesungen über die Psychoanalyse de 1909. Celle-ci, due à Yves Le Lay, a été publiée en trois parties dans La Revue de Genève, en décembre $1920^{37}$ (avec une présentation d'Édouard Claparède, intitulée «Freud et la psychanalyse » ${ }^{38}$ ), en janvier 1921 (p. 80-87) et en

33. - Dans la préface de Félix Bertaux à son Panorama de la littérature allemande contemporaine, Paris, Kra, 1928.

34. - Cité in Karl Zieger, op. cit., p. 223.

35. - Jean-Pierre Meylan, La Revue de Genève, miroir des lettres européennes, op. cit., p. 125.

36. - Élisabeth Roudinesco, Histoire de la psychanalyse en France (Paris, Fayard, 1994), cité d'après l'édition Pochothèque, Le Livre de poche, Paris, Librairie générale française, 2009, p. 669.

37. - La Revue de Genève, 1920, nº (décembre), p. 865-875.

38. - Ibid., p. 846-864. Dans le numéro précédent (La Revue de Genève, 1920, n5 (novembre), p. 729-737, était publié un article de Joseph Redlich intitulé «Autriche. I- Un peuple qui agonise » d'une tonalité très pessimiste («Ce qui se passe sous nos yeux à Vienne et dans les autres villes d'Autriche, c'est une lente et irrésistible désagrégation, l'écroulement d'un peuple tout entier et d'une grande et ancienne culture »). 
février 1921 (p. 195-220) sous le titre « Origine et développement de la psychanalyse ». En 1925, la revue publie encore «La psychanalyse et l'art » de L. Charles-Baudoin ${ }^{39}$.

Ainsi la Suisse romande et La Revue de Genève ont confirmé la déclaration de Freud dans Contribution à l'histoire du mouvement psychanalytique (1914):

C'est dans la Suisse intellectuellement si active qu'affluent les représentants des nations les plus importantes; un foyer d'infection en ce lieu ne pouvait qu'être particulièrement important pour la propagation de l'épidémie psychique, comme Alfred Hoche l'avait qualifiée à Fribourg 40 .

Un peu avant, en 1913, Alfons Maeder avait estimé que « c'est de la Suisse romande seule que doit se faire, un jour, la vulgarisation de la psychanalyse en pays latins. [...] Nulle part le terrain n'est mieux préparé; la France n'est pas mûre du tout pour la psychanalyse $»^{41}$. Ainsi, sous la plume de Félix Bertaux, l'évocation de la « vibration freudienne » caractéristique de Schnitzler était-elle, en effet, ambivalente: à Paris, nombreux sont, à l'époque, ceux qui taxent la psychanalyse freudienne de réductionnisme pansexualiste et qui prétendent qu'elle serait l'émanation délétère d'un genius loci viennois décadent. Dans sa préface à La Pénombre des âmes de Schnitzler, Félix Bertaux ne parle pas de vibration, mais de teinte freudienne: "Pour l'étude des complications du cœur, la psychiatrie venait au secours de la psychologie. L'analyse devenait psychanalyse. Le $\mathrm{D}^{\mathrm{r}}$ Freud était à la mode et les personnages de Schnitzler sont teintés de freudisme ${ }^{42}$. Dans la présentation du Célibataire, Félix Bertaux explique encore: "Schnitzler, médecin et psychologue, n'a pas échappé à l'influence de Freud; sa psychologie est de la psychopathie. Sans rien pourtant qui sente la théorie, ni le travail de laboratoire ${ }^{43}$. Vibration, teinte, psychologie, psychopathie,

39. - Bibliothèque universelle et Revue de Genève, 1925, vol. 1 (janvier-juin), p. 305-314.

40. - Sigmund Freud, Contribution à l'histoire du mouvement psychanalytique, trad. Pierre Cotet et René Lainé, in S. Freud, Euvres complètes. Psychanalyse, éd. André Bourguignon, Pierre Cotet, Jean Laplanche, vol. XII : 1913-1914, Paris, PUF, 2005, p. 270.

41. - Élisabeth Roudinesco, Histoire de la psychanalyse en France, op. cit., p. 357.

42. - Préface de Félix Bertaux à Schnitzler, La Pénombre des âmes, Paris, Stock, 1929, citation in Karl Zieger, Enquête sur une réception. Arthur Schnitzler et la France..., op. cit., p. 204.

43. - Présentation de Félix Bertaux, A. Schnitzler, «Le Célibataire », in Romanciers allemands. Nouvelles de Gerhart Hauptmann, Arthur Schnitzler, etc., trad. sous la direction de Félix Bertaux, préface d'Edmond Jaloux, Paris, Denoël et Steele, 1932, citation in Karl Zieger, op. cit., ibid. 
laboratoire: ces approximations sont fort inexactes et superficielles et l'on sent bien que Félix Bertaux prend avec des pincettes le freudisme qu'il prétend avoir repéré chez Schnitzler, suggérant que c'est surtout en évitant que ses textes « sentent la théorie » que l'auteur viennois a fait la preuve de son talent. Aux yeux de Félix Bertaux, ce « freudisme » pourrait bien constituer un obstacle à l'introduction de Schnitzler en France. On a le sentiment qu'il cherche à rassurer le lecteur français en soulignant que Schnitzler est avant tout un fin connaisseur de l'âme humaine.

En revanche, dans le contexte de La Revue de Genève, même sous la plume du Français Daniel-Rops, le rapprochement de Schnitzler et de Freud a la valeur d'un éloge sans ambiguïté. S'il est bien vrai que Daniel-Rops lui-même ne va pas au-delà d'une citation des propos de Félix Bertaux, pour Robert de Traz, le «freudisme » est une promesse de renouvellement du roman d'analyse psychologique.

Dans d'autres contextes, il est vrai, l'évocation de Freud intervient sur le ton du horresco referens. Ainsi Emmanuel Buenzod présente en 1927 la traduction de Hermann Ungar, Enfants et meurtrier, publiée aux Éditions de la NRF, en ces termes:

Deux sombres nouvelles. Elles nous viennent de Tchécoslovaquie et ne sont pas sans révéler l'influence des théories de Freud sur l'esprit de leur auteur. L'horreur et la démence rôdent dans ces pages. [...] La funèbre lecture! Est-il bien nécessaire d'exporter de si désolants produits ? 44

Il serait exagéré d'affirmer que La Revue de Genève a joué un rôle de premier plan dans la réception de Schnitzler en France et en Suisse. Ni la traduction due à Geneviève Maury, ni le compte rendu de DanielRops n'ont eu un retentissement exceptionnel. C'est le contexte de $L a$ Revue de Genève qui retient l'attention: dans cette revue à la fois européenne et attachée à la défense et illustration de « l'esprit de Genève », à la fois conservatrice dans ses options esthétiques et idéologiques et audacieuse lorsqu'elle apporte son soutien à Freud et à la psychanalyse, l'importance accordée à Schnitzler prend un sens particulier. «L'esprit de Genève » dialogue avec « l'esprit viennois ».

44. - Bibliothèque universelle et Revue de Genève, 1927, vol. 1 (janvier-juin), p. 686. 